\title{
ASSOCIATION OF HbA1C, FASTING GLUCOSE LEVELS AND LIPID PARAMETERS WITH ANTHROPOMETRIC INDICES IN A GROUP OF DIABETICS AND NON-DIABETIC INDIVIDUALS
}

\author{
Burcu Dogan ${ }^{1}$, Can Öner²
}

\author{
${ }^{1}$ Sakarya Training and Research Hospital, Department of Family Medicine, Sakarya, Turkey \\ ${ }^{2}$ Kartal Dr Lutfi Kirdar Training and Research Hospital, Department of Family Medicine, Istanbul, Turkey
}

\begin{abstract}
Objective: Because of the usefulness of anthropometric measurements and indices, they are frequently recommended for prediction of diabetes in clinical practice. The aim of this study is to determine the relationship between HbA1c and fasting plasma glucose (FPG) and lipid parameters of diabetics and non-diabetics and 7 different indices, such as, waist circumference (WC), waist-to-hip ratio (WtHR), body mass index (BMI), Broca-Katsura index, Rohrer index, a body shape index (ABSI), and body roundness index (BRI).
\end{abstract}

Methods: A total of 505 individuals were included in the study (type 2 DM, n=65; type 1 DM, n=265, and 171 controls). Anthropometric measurements were evaluated (weight, height and waist circumference) and from these measurements BMI, Broca-Katsura Index, Rohrer Index, ABSI and BRI were calculated. HbA1c, FPG, LDL-cholesterol, triglyceride and HDL-cholesterol levels were also measured. Data were analysed with SPSS 21.0 statistics program.

Results: HbA1c levels and BMIs were significantly high in diabetic patients. None of the indices were significantly correlated with HbA1c, FPG levels, LDL-cholesterol and triglyceride levels in diabetic patients; but body weight, body mass, BMI, WC and BrocaKatsura index were positively correlated with HbA1c levels in controls. On the other hand, FPG levels of controls were positively correlated with body weight, BMI and Broca-Katsura index.

Conclusion: Although much of the anthropometric measurements and indices correlated with the levels of $H b A 1 c, F P G$ and $L D L-$ cholesterol, HDL-cholesterol and triglycerides in control group; none of the indices were correlated with HbA1c levels, FPG, LDLcholesterol and triglyceride in type 1 and type 2 diabetics. Anthropometric indices, especially recent ones, had limited capacity and usefulness for monitoring of diabetes and dyslipidemia in diabetic patients.

Key words: Type 1 diabetes, type 2 diabetes, body mass index, anthropometric measurements.

\section{Introduction}

Obesity and diabetes mellitus are two important public health problems throughout the world. Obesity, especially central obesity, is strongly and directly associated with development of diabetes mellitus (1). It was shown many times that diabetes acts as a vascular disease and if it was uncontrolled, can cause micro and macro vascular diseases. Several studies emphasize the importance of intensive glucose control in diabetic patients $(2,3)$. Control of obesity results in decrease of $\mathrm{HbA1c}$ levels and can help control of diabetes.

\begin{abstract}
There are many anthropometric measures and indices used for prediction of obesity. The most frequently used and known index is body mass index (BMI) (4). Other than BMI, waist circumference (WC), waist-to-height ratio (WHtR) and waist-to-hip ratio (WHpR) also used for prediction of obesity and especially central obesity (5). These direct anthropometric measurements have some limitations. BMI focuses on weight and enables to distinguish fat and muscle mass. On the other hand, WC reflects only abdominal fat accumulation and dismisses total body composition (6).
\end{abstract}


Table 2. Clinical characteristics of participants

\begin{tabular}{|c|c|c|c|c|}
\hline & $\begin{array}{c}\text { Type 1 DM } \\
(\mathbf{n = 2 6 5 )}\end{array}$ & $\begin{array}{c}\text { Type 2 DM } \\
(\mathbf{n = 6 5 )}\end{array}$ & $\begin{array}{c}\text { Control } \\
(\mathbf{n = 1 7 1 )}\end{array}$ & $\boldsymbol{p}$ \\
\hline $\begin{array}{c}\text { Gender } \\
\text { Female }\end{array}$ & $\begin{array}{c}141(53.21 \%) \\
124(46.79 \%)\end{array}$ & $\begin{array}{c}40(61.54 \%) \\
25(38.46 \%)\end{array}$ & $\begin{array}{c}70(40.94 \%) \\
101(59.06 \%)\end{array}$ & 0.006 \\
\hline Age (years) & $36.5 \pm 10.8$ & $38.2 \pm 9.2$ & $37.6 \pm 11.4$ & 0.397 \\
\hline BMI (kg/m $\left.{ }^{2}\right)$ & $24.7 \pm 5.3$ & $29.5 \pm 5.0$ & $26.0 \pm 4.8$ & $<0.001$ \\
\hline FPG (mg/dL) & $199.3 \pm 99.4$ & $173.2 \pm 65.9$ & $89.9 \pm 11.9$ & $<0.001$ \\
\hline HbA1c (\%) & $8.6 \pm 1.9$ & $8.3 \pm 2.2$ & $5.4 \pm 0.2$ & $<0.001$ \\
\hline
\end{tabular}

BMI: Body Mass Index; FPG: Fasting Plasma Glucose; HbA1c: Glycated Hemoglobin

Data are presented as $n(\%)$ or median (minimum-maximum), where appropriate.

Table 3. The correlation of $\mathrm{HbA1c}$ with different indices in three groups

\begin{tabular}{|c|c|c|c|c|}
\hline \multicolumn{2}{|c|}{ Anthropometric measures and indices } & \multirow{2}{*}{$\begin{array}{c}\text { Type } 1 \text { DM } \\
\begin{array}{c}(n=265) \\
-0.024\end{array}\end{array}$} & \multirow{2}{*}{$\begin{array}{c}\text { Type } 2 \text { DM } \\
\begin{array}{c}(n=65) \\
0.095\end{array}\end{array}$} & \multirow{2}{*}{$\begin{array}{c}\text { Control } \\
\text { ( } n=171)\end{array}$} \\
\hline Whaight (kat) & $\mathrm{r}$ & & & \\
\hline VveIgnt (KE) & $\mathrm{p}$ & 0.690 & 0.450 & $<0.001$ \\
\hline \multirow{2}{*}{ BMI $\left(\mathrm{kg} / \mathrm{m}^{2}\right)$} & $\mathrm{r}$ & -0.080 & 0,013 & 0.295 \\
\hline & $\mathrm{p}$ & 0.890 & 0.911 & $<0.001$ \\
\hline \multirow{2}{*}{ Waist circumference $(\mathrm{cm})$} & $\mathrm{r}$ & -0.027 & 0.014 & 0.323 \\
\hline & $\mathrm{p}$ & 0.658 & 0.911 & $<0.001$ \\
\hline \multirow{2}{*}{ Waist/ Height } & $\mathrm{r}$ & -0.033 & 0.026 & -0.137 \\
\hline & $\mathrm{p}$ & 0.601 & 0.840 & 0.075 \\
\hline \multirow{2}{*}{ Broca-Katsura Index } & $\mathrm{r}$ & -0.030 & 0.023 & 0.314 \\
\hline & $\mathrm{p}$ & 0.630 & 0.855 & $<0.001$ \\
\hline \multirow{2}{*}{ Rohrer Index } & $\mathrm{r}$ & -0.025 & 0.002 & -0.144 \\
\hline & $\mathrm{p}$ & 0.690 & 0.989 & 0.062 \\
\hline \multirow{2}{*}{ ABSI } & $r$ & -0.013 & -0.074 & 0.094 \\
\hline & $\mathrm{p}$ & 0.835 & 0.564 & 0.227 \\
\hline \multirow{2}{*}{ BRI } & r & 0.001 & -0.021 & 0.100 \\
\hline & $\mathrm{p}$ & 0.992 & 0.871 & 0.193 \\
\hline
\end{tabular}

ABSI: A Body Shape Index; BMI: Body Mass Index; BRI: Body Roundness Index, DM: Diabetes Mellitus

Pearson correlation test 
significantly correlated with $\mathrm{HDL}$-cholesterol levels namely; weight $(r=-0.302 ; p<0.001)$, WC $(r=-0.310 ; p<0.001)$, BMI $(r=-$ 0.175; $p=0.04)$, WtHR ( $r=0.199 ; p=0.009)$, Broca-Katsura Index $(r=0.242 ; p=0.002) ; A B S I(r=0.191 ; p=0.013)$. LDLcholesterol levels of control groups were significantly correlated with weight $(r=0,216 ; p<0.001)$; WC $(r=0.296$; $p<0.001)$; BMI $(r=0.252 ; p=0.001) ; A B S I(r=0.191 ; p=0.013)$ and Broca-Katsura Index $(r=0.241 ; p=0.002)$. Moreover, triglyceride levels of control group also significantly correlated with weight $(r=0.422 ; p<0.001)$; WC $(r=0.388$; $p<0.001)$; BMI $(r=0.336 ; p<0.001) ; A B S I(r=-0.317 ; p<0.001)$ and Broca-Katsura Index ( $r=-0.222 ; p=0.002)$.

\section{Discussion}

We found that all of the anthropometric indices do not correlate with $\mathrm{HbA1c}$ levels in diabetics; whereas weight, WC, BMI were positively associated with HbA1c levels in controls. The levels of $\mathrm{HbA} 1 \mathrm{c}$ are valuable measurements for monitoring glucose levels over the proceeding 2-3 months and therefore it is useful for evaluation of clinical monitoring of diabetic patients (10).

Because of the simpleness and usefulness of weight, waist circumference and BMI, they have been recommended for prediction of diabetes in clinical practice (11). Hartwig et al. found that weight, waist circumference and weight-toheight ratio were anthropometric markers of type 2 diabetes. Among them WtHR is the strongest predictor of type 2 diabetics (12). In another study conducted in China with 11687 type 2 diabetics, it was found that increased BMI, WC, WHtR and ABSI values were related with the incidence of type 2 DM. But after adjustment of multicovariates, it was shown that the risk of type 2 diabetes increased with elevated $A B S I$, but not differs with increase of BMI, WC and WtHR (13). However, BMI has been criticized especially for the discrimination of fat, lean body mass and fat distribution (14). Therefore, new anthropometric indices were proposed as alternatives, $A B S I$ and BRI. But it was shown that neither ABSI nor BRI were superior to $B M I, W C$, or $W H t R$ for predicting the presence of DM. ABSI showed the weakest predictive ability, while BRI showed potential for use as an alternative obesity measure in the assessment of DM (15). We found that HDL-cholesterol levels were significantly correlated with weight, WC, and BMI in three groups, but LDL-cholesterol and triglyceride levels of diabetics do not correlated with any measurements and indices. On the other hand, all of the lipid parameters were significantly correlated with weight, WC, BMI, ABSI and Broca-Katsura Index in control group.

Table 4. The correlation of fasting plasma glucose with different indices in three groups

\begin{tabular}{|c|c|c|c|c|}
\hline \multicolumn{2}{|c|}{ Anthropometric measures and indices } & \multirow{2}{*}{$\begin{array}{c}\begin{array}{c}\text { Type } 1 \text { DM } \\
\text { (n=265) }\end{array} \\
0.035\end{array}$} & \multirow{2}{*}{$\begin{array}{c}\text { Type 2 DM } \\
(n=65)\end{array}$} & \multirow{2}{*}{$\begin{array}{c}\begin{array}{c}\text { Control } \\
(\mathbf{n}=171)\end{array} \\
0.234\end{array}$} \\
\hline & $r$ & & & \\
\hline (1) & $\mathrm{p}$ & 0.576 & 0.839 & 0.002 \\
\hline \multirow{2}{*}{ BMI $\left(\mathrm{kg} / \mathrm{m}^{2}\right)$} & r & 0.056 & -0.056 & 0.217 \\
\hline & $\mathrm{p}$ & 0.370 & 0.662 & 0.005 \\
\hline \multirow{2}{*}{ Waist circumference $(\mathrm{cm})$} & $\mathrm{r}$ & 0.083 & -0.075 & -0.047 \\
\hline & $\mathrm{p}$ & 0.181 & 0.555 & 0.544 \\
\hline \multirow{2}{*}{ Waist/Height } & $r$ & 0.092 & -0.060 & -0.047 \\
\hline & $\mathrm{p}$ & 0.134 & 0.640 & 0.544 \\
\hline \multirow{2}{*}{ Broca-Katsura Index } & $r$ & 0.058 & -0.036 & 0.209 \\
\hline & $\mathrm{p}$ & 0.354 & 0.735 & 0.006 \\
\hline \multirow{2}{*}{ Rohrer Index } & $\mathrm{r}$ & 0.056 & -0.051 & -0.051 \\
\hline & $\mathrm{p}$ & 0.368 & 0.656 & 0.511 \\
\hline \multirow{2}{*}{ ABSI } & $\mathrm{r}$ & 0.016 & -0.041 & 0,052 \\
\hline & $\mathrm{p}$ & 0.800 & 0.742 & 0,952 \\
\hline \multirow{2}{*}{ BRI } & $\mathrm{r}$ & 0.028 & -0.056 & -0.051 \\
\hline & $\mathrm{p}$ & 0.600 & 0.657 & 0.511 \\
\hline
\end{tabular}

BMI: Body Mass Index; ABSI: A Body Shape Index; BRI: Body Roundness Index, DM: Diabetes Mellitus

Pearson correlation test 
In a study from Korea with the sample size of 1032 adults, it was shown that lipid parameters were significantly correlated with weight, WC, WtHR and BMI in women. BMI was not significantly correlated with lipid parameters in men (16). It was shown that ABSI was significantly correlated with LDL-cholesterol and triglycerides levels in healthy young adult men (17). Zaid et al. compared recently described indices, $A B S I$ and $B R I$ for assessment capacity of type and severity of dyslipidemia. As a result, they found that the dyslipidemia prediction capacity of BRI was comparable, but not superior to BMI and WC. On the other hand, the ABSI could not detect the presence or absence of dyslipidemia (18).

In conclusion, although much of the anthropometric measurements and indices correlated with $\mathrm{HbA1c}$ levels, FPG, LDL-cholesterol, HDL-cholesterol and triglycerides levels in healthy controls, none of the indices were correlated with HbA1c levels, FPG, LDL-cholesterol and triglyceride in type 1 and type 2 diabetics. But HDLcholesterol levels were associated with conventional measurements in diabetics. So anthropometric indices, especially recent ones, has limited capacity and usefulness for monitoring of diabetes and dyslipidemia in diabetic patients.

\section{Conflict of interest: None.}

Funding source: There is no funding in this article.

\section{References}

1. Gharipour $M$, Sadeghi $M$, Danetkhah $M$, Bidmeshgi $S$, Ahmadi A, Tahri $M$ et al. The cut-off values of anthropometric indices for identifying subjects at risk for metabolic syndrome in Iranian elderly men. J Obes 2014;2014:907149.

2. The Diabetes Control and Complications Trial Research Group. The effect of intensive treatment of diabetes on the development and progression of long-term complications in insulin-dependent diabetes mellitus. $\mathrm{N}$ Engl J Med 1993;329:977-86.

3. UK Prospective Diabetes Study (UKPDS) Group. Intensive blood-glucose control with sulphonylureas or insulin compared with conventional treatment and risk of complications in patients with type 2 diabetes (UKPDS 33). Lancet 1998;352:837-53.

4. World Health Organization. Physical status: The use and interpretation of anthropometry. Technical Report Series 854, Geneva, 1995.

5. Gu Z, Li D, He H, Wang J, Hu X, Zhang P et al. Body mass index, waist circumference and waist to height ratio for prediction of multi metabolic risk factors in Chinese elderly population. Scientific Reports 2018;8(1):385.
6. Fujita M, Sato $Y$, Nagashima K, Takahashi S, Hata A. Predictive power of a body shape index for development of diabetes, hypertension, and dyslipidemia in Japanese adults: A retrospective cohort study. PLoSOne 2015;10(6):e0128972.

7. Bergman NR, Stefanovski D, Buchanan $T A$, Summer $A E$, Reynolds JC, Sebring NG et al. A beter index of body adiposity. Obesity(Silver Spring) 2011;19(5):1083-9.

8. Krakauer NY, Krakauer JC. A new body shape index predicts mortality hazard independently of body mass index. PLoSONE 2012;7(7):e39504.

9. Thomas DM, Bredlau C, Bosy-Westphal A, Mueller M, Shen W, Gallagher $D$ et al. Relationships between body roundness with body fat and visceral adipose tissue emerging from a new geometrical model. Obesity(Silver Spring) 2013;21(11):2264-71.

10. Koga M, Matsumoto $S$, Saito $H$, Kasayama S. Body mass index negatively influences glycated albumin, but not glycatedhemoglobin, in diabetic patients. Endocr J 2006;53(3):387-91.

11. Qiao $Q$, Nyamdorj R. Is the association of type II diabetes with waist circumference or waist-to-hip ratio stronger than that with body mass index? Eur J ClinNutr 2010;64:30-4.

12. Hartwig S, Kluttig A, Tiller D, Fricke J, Müller G, Schipf S et al. Anthropometric markers and their association with incident type 2 diabetes mellitus: which marker is best for prediction? Pooled analysis off our German population based cohort studies and comparison with a nationwide cohort study. BMJ Open 2016;6:e009266.

13. Han C, Liu Y, Sun X, Luo X, Zhang L, Wang B et al. Prediciton of new body shape index and body adiposity estimator for development of type 2 diaetes mellitus: The rural Chinese cohort study. Br J Nutr 2017;118(10);771-6.

14. Heymsfield SB, Scherzer R, Pietrobelli A, Lewis CE, Grunfeld $C$. Body mass index as a phenotypic expression of adiposity: quantitative contribution of muscularity in a population-based sample. Int J Obes(Lond) 2009;33:136373.

15. Chang Y, Guo X, Chen Y, Guo L, Li Z, Yu S et al. A body shape index and body roundness index: two new body indices to identify diabetes mellitus among rural populations in northeast China. BMC Public Health 2015; 15:794.

16. Jeong SK, Seo MW, Kim YH, Kweon SS, Nam HS. Does waist indicate dyslipidemia better than BMI in Korean adult population. J Korean Med Sci 2005;20(1):7-12.

17. Malara M, Keska A, Tkaczyk J, Lutoslawska G. Body shape index versus body mass index as correlates of health risk in young healthy sedentary men. J Transl Med 2015;13:75.

18. Zaid M, Ameer F, Munir R, Rashid R, Farooq N, Hasnain S et al. Anthropometric and metabolic indices in assessment of type and severity of dyslipidemia. J Physiol Anthropol 2017;36:19. 